\title{
Can we prevent seasonal affective disorder (SAD) with melatonergic agents? ${ }^{\dagger}$
}

\author{
Tarek Zghoul (1)
}

\begin{abstract}
SUMMARY
Seasonal affective disorder (SAD) is a recurrent form of major depression, particularly occurring in the winter months with a generally spontaneous remission in spring/summer. The predictable nature of this condition provides a potentially unique opportunity to prevent recurrence in sufferers of SAD. The Cochrane Review discussed here examines the evidence for melatonin and agomelatine in preventing SAD, putting its findings into their clinical context.
\end{abstract}

\section{KEYWORDS}

Antidepressants; circadian rhythms; seasonal affective disorder; agomelatine; melatonin.

Seasonal affective disorder (SAD) is a form of depression with a seasonal pattern. It occurs particularly in the winter, with subsequent remission in the spring or summer, and displays a latitude characteristic, with a higher prevalence in northern latitudes (Monteleone 2008). In the UK, it has been estimated that $5.6 \%$ of patients attending general practices in the winter suffer from $\mathrm{SAD}$ (Thompson 2004).

ICD-10 classifies SAD as a subtype of major depressive disorder characterised by typical (e.g. lowered mood, anhedonia and anergia) but also atypical (hypersomnia, carbohydrate craving and weight gain) depressive symptoms with a characteristic seasonal nature (Rosenthal 1984; Magnusson 2005; WHO 1992). As with many other psychiatric disorders, $\mathrm{SAD}$ is a complex condition in which a multitude of genetic and physical environmental factors contribute to its manifestation. These include chronobiological mechanisms related to circadian rhythms, photoperiodism, melatonin and major monoamine neurotransmitters such as serotonin and dopamine (Levitan 2007; Lewy 2009). More generally, disrupted chronobiological mechanisms have been linked to major depressive disorder and, importantly, some of the more efficacious treatments of this disorder are therapies actively targeting the circadian system, such as morning bright light and wake therapy (Walker 2020). SAD is a relatively stable condition in which two-thirds of patients continue to display symptoms in the long term, with only $20 \%$ demonstrating complete remission within a few years following first diagnosis (BMJ 2009).

As regards treatment, the National Institute for Health and Care Excellence (NICE; 2009) does not recommend treating seasonal depression any differently from major depressive disorder. No particular antidepressant is favoured and light therapy is also not actively encouraged (NICE cites a lack of evidence). However, findings have suggested that melatonin, a rhythm-regulating hormone, and circadian rhythms may play a role in $\mathrm{SAD}$, with melatonin exhibiting chronobiotic properties and the ability to induce phase shifts (Hickie 2011). In this context, the antidepressant agomelatine, which was approved by the European Medicines Agency (EMA) in 2009 for the treatment of depression and has subsequently been demonstrated to be one of the more effective antidepressant agents (Cipriani $2018)$, is a melatonin receptor agonist $\left(\mathrm{MT}_{1}\right.$ and $\left.\mathrm{MT}_{2}\right)$ and serotonin receptor antagonist $\left(5-\mathrm{HT}_{2} \mathrm{C}\right.$ and $\left.5-\mathrm{HT}_{2} \mathrm{~B}\right)$, and has been shown to restore disrupted circadian rhythms as well as being effective in treating people with SAD (Pjrek 2007; Srinivasan 2012). Thus, given the role of melatonergic systems on a neurochemical level in $\mathrm{SAD}$, targeting these systems with melatonergic agents with the aim of preventing SAD appears reasonable. Further, with the predictable seasonal pattern of SAD arises a hopeful opportunity for prevention. The authors of this month's Cochrane Corner (Nussbaumer-Streit 2019a) thus wished to review the efficacy and safety of melatonin and agomelatine in preventing $\mathrm{SAD}$, as this had not been previously accomplished.

\section{The Cochrane Review}

The review authors found only one study that met their eligibility criteria, and this compared agomelatine with placebo, providing data on 225 adults with a history of winter-type SAD who were symptom free at the beginning of the study. The analysis
ROUND THE CORNER
Tarek Zghoul, PhD, MRCPsych, is a specialty registrar in general adult psychiatry with Oxford Health NHS Foundation Trust and a Senior Research Fellow in the Department of Psychiatry, Warneford Hospital, University of Oxford, UK. He works with cognitive neuropsychological models in the area of depression. Correspondence Tarek Zghoul. Email: tarek.zghoul@psych.ox.ac.uk

First received 25 Feb 2020 Final revision 30 Mar 2020 Accepted 6 Apr 2020

Copyright and usage The Author 2020

${ }^{\dagger}$ Commentary on... Melatonin and agomelatine for preventing seasonal affective disorder: a Cochrane Review. See this issue. 
demonstrated inconclusive results, showing no clear effect either in favour or against agomelatine as a preventive treatment in SAD. Owing to the absence of studies on melatonin and the unclear evidence on agomelatine, no conclusion about the safety and efficacy of melatonin and agomelatine in the prevention of SAD may currently be inferred.

\section{Definition of the clinical question}

The review aimed to assess, in a population of adults with a history of SAD, the safety and efficacy of melatonin and agomelatine, whether in comparison with each other, placebo and other interventions (e.g. psychological and light therapy), in preventing a repeat onset of $\mathrm{SAD}$.

A population of 225 participants over 18 years of age with a history of SAD but not displaying a current depressive episode at the start of the intervention were included. The sample was wholly derived from one study. SAD was defined in line with DSM-5 criteria as a seasonal pattern of recurrent major depressive episodes (American Psychiatric Association 2013). The definition, however, was then further restricted to the wintertype form, which is characterised by the depressive episode occurring in either the autumn or winter and then experiencing a full remission in either spring or summer. Summer-type SAD presents with different symptomatology and is a rarer form (Akram 2019). Participants with bipolar affective disorder were excluded, as were people demonstrating a depressive disorder from another medical condition, but studies that included people at risk of $\mathrm{SAD}$ and whose medical condition was not the direct cause for their depressive episode were considered. The sample, however, would have been increased had a population with bipolar disorder been included as well: it is estimated that around $25 \%$ of people with bipolar disorder fulfil criteria for SAD (Geoffroy 2013).

To assess efficacy, Nussbaumer-Streit et al considered only randomised controlled trials (RCTs), but for adverse events (i.e. tolerability) the intention was to include non-RCTs as well. The primary outcome measures were in relation to benefit and harm. Benefit was measured by the incidence of $\mathrm{SAD}$. This was measured using the Structured Interview Guide for the Hamilton Depression Rating Scale - Seasonal Affective Disorders version (SIGH-SAD) with a score set a priori by the review authors at $\geq 20$ or as defined by study authors themselves. The primary outcome for harm was defined as the rate of adverse events in relation to the intervention(s) employed (i.e. tolerability). The review authors also intended to study several secondary outcome measures, such as quality of life and interpersonal and social functioning, the severity of the $\mathrm{SAD}$ episode, the proportion of people with serious adverse events and discontinuation rates (i.e. acceptability).

\section{Methods}

The search strategy included a review of multiple electronic databases as well as grey literature, including the European Medicines Agency (EMA), with no restrictions as to date, language or publication status. The references of identified studies were then scrutinised for any further relevant studies. Subject experts and trialists were contacted for information about ongoing or unpublished studies or to request additional trial data. In the case of the included study (which was unpublished), comprehensive data were obtained from the EMA. Two review authors independently reviewed the citations yielded by the search and, if required, resolved disagreements by consulting a third party.

The search initially returned 3745 citations but only one study, comparing agomelatine ( $25 \mathrm{mg} /$ day) with placebo, met the set eligibility criteria. Specifically, of the 3745 records, 3619 were excluded during title and abstract review, leaving 126 titles for full text review and assessment for eligibility. Of these, 125 records were then rightly excluded for the following reasons: ineligible populations (54), ineligible publications such as editorials (25), ineligible interventions/controls (14), ineligible study designs (21), 'does not answer a question of the review' (3), conference abstracts only (2), and already included in other Cochrane Reviews on $\mathrm{SAD}$ - such as the review on light therapy (Nussbaumer-Streit 2019b) - and not relevant for inclusion in this review (6). Those studies that narrowly missed the inclusion criteria were rightly excluded for the following reasons: participants did not meet the criteria for $\mathrm{SAD}$ and were suffering from another form of depressive disorder, or participants were already displaying depressive symptoms at the start of the study. The review authors included only RCTs, in accordance with their inclusion criteria, and 21 studies were excluded owing to ineligible study designs. Potentially, and if available, non-randomised studies such as cohort studies and case-control studies could have been included in this review, with the 'Risk of Bias in Non-randomised Studies - of Interventions' (ROBINS-I) tool applied for assessing risk of bias in these non-RCTs.

Certainty of the evidence was assessed using the GRADE approach (Schünemann 2013). Risk of bias (Box 1 and Fig. 1) was assessed by two review authors using the Cochrane risk-of-bias tool as outlined in Higgins et al (2019). The review authors specifically assessed attrition and the reasons for it, 
BOX 1 Bias, attrition bias and validity

A bias is a systematic error in results or inferences and can be defined as 'any process at any stage of inference which tends to produce results or conclusions that differ (systematically) from the truth' (Sackett 1979). It is systematic in that multiple replications of the same study would on average lead to the wrong answer.

Attrition bias can occur when people drop out of a study, leading to systematic differences between participants who leave the study and those who continue. This, in effect, can then weaken internal and external validity. as well as whether all relevant outcomes in these trials were published.

The statistical analysis of data used intention to treat (ITT) (Box 2) and risk ratios (RRs) (Box 3) as effect sizes for dichotomous data and mean difference (MD) for continuous data, all with 95\% confidence intervals (CIs).

\section{Results}

Only one trial (225 participants) provided data for the outcomes presented in the review. Regarding the primary outcomes and the incidence of SAD, 199 participants were part of the analysis set. These were participants who had taken at least one dose of the intervention (agomelatine) and had at least one follow-up visit (26 people were excluded on this basis). The results were inconclusive, with wide confidence intervals that may encompass both relevant beneficial as well as harmful effects of agomelatine $(\mathrm{RR}=0.83,95 \%$ CI $0.51-1.34)$.

\section{BOX 2 Intention-to-treat analysis}

Intention-to-treat (ITT) analysis is the gold standard for randomised clinical trials. Under the ITT principle, participants taking part in the study are analysed as members of the group to which they were initially randomised, irrespective of whether they left the study, adhered to their treatment or switched to another one. IT analyses are frequently used to assess clinical effectiveness as they mirror actual clinical practice, where adherence to the treatment is not always followed or where the treatment has to be changed according to the patient's clinical response.
Assessing the impact of the excluded 26 participants, best- and worst-case scenarios (Box 4) were calculated, with the former yielding significance: best-case scenario (assuming that no one who dropped out was depressed) $(\mathrm{RR}=0.42,95 \% \mathrm{CI}$ 0.18-0.96), worst-case scenario (assuming that everyone who dropped out was depressed) ( $\mathrm{RR}=0.96,95 \%$ CI 0.66-1.38).

Regarding adverse events, 64 out of 112 people in the agomelatine group and 61 out of 113 in the placebo group displayed adverse events $(\mathrm{RR}=$ 1.06, 95\% CI 0.84-1.34). The overall discontinuation rate saw 49 people in the agomelatine and 59 people in the placebo group leave the study $(\mathrm{RR}=0.84,95 \%$ CI 0.64-1.10).

Regarding secondary outcomes, the severity of $\mathrm{SAD}$ may be similar in both groups $(\mathrm{MD}=-1.80$, $95 \%$ CI -4.58 to 0.98 ). No data were available for quality of life or interpersonal and social functioning.

The certainty of evidence for all outcomes was rated as very low because of high risk of bias, imprecision and indirectness.

Random sequence generation (selection bias)

Allocation concealment (selection bias)

Masking of participants and personnel (performance bias)

Masking of outcome assessment (detection bias

Incomplete outcome data (attrition bias)

Selective outcome reporting (reporting bias)

Other bias

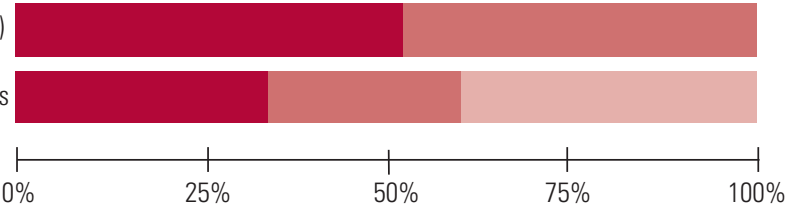


BOX 3 Risk ratio

Risk ratio (RR) can be understood as a measure of the risk of an event occuring in the intervention group compared with the risk of the same event occuring in the control group.

$\mathrm{RR}=1$ means that there is no difference between the groups.

$R R<1$ means that the risk of the event occuring has been reduced by the intervention.

$\mathrm{RR}>1$ means that the risk of the event occurring in the intervention group has been increased.

\section{Discussion}

In summary, this Cochrane Review did not demonstrate a beneficial role for agomelatine or melatonin in the prevention of SAD. It was based on only one study and was therefore in effect an appraisal of a single study. Although there is a good amount of research into the treatment of SAD using melatonergic agents, with some studies demonstrating their ameliorative effects, there is a marked lack of research into the actual prevention of $\mathrm{SAD}$ in the first place. This may be due to a lack of interest but also, importantly, due to the complexity of conducting such studies, especially in the recruitment of known individuals with $\mathrm{SAD}$ who are symptom free at the start of such an investigation.

\section{The evidence on melatonergic agents}

In the case of agomelatine, the evidence was uncertain and of very low quality. In the case of melatonin, a drug readily available over the counter in many countries, the review authors found no studies at all, and this may be a result of an inability to attain financing for such studies from bodies such as the pharmaceutical industry. In this context, the included agomelatine study was funded by the pharmaceutical industry.

BOX 4 Best-case and worst-case scenario

Missing outcome data are a frequent problem in systematic reviews, which may affect the validity of the study. The best-case scenario assumes that all missing participants have favourable outcomes in the intervention group and poor outcomes in the control group. The worst-case scenario assumes the opposite.

These scenarios are generally used as a sensitivity analysis and may generate unrealistic results in practice, in particular if attrition rates are elevated.
The review authors employed rigorous but necessary inclusion and exclusion criteria such as in differentiating participants with actual SAD from those with other depressive disorders and those who were symptom free at the start of the study from those who were already presenting with symptoms. In the end, only one study was included in the review. This 2008 study by Kasper et al, on the efficacy and safety of S 20098 (agomelatine) administered once a day for 1 year after an open period of at least 18 weeks, had not been published in a scientific journal and the review authors retrieved the complete trial data from the EMA, which is a credit to them.

The study itself suffered from a very high attrition rate $(45 \%$ in the agomelatine group; $52 \%$ in the placebo group), which affected the results' validity (Box 1). The dose of agomelatine (25 mg) employed may have been appropriate but perhaps a higher dose may have seen a more beneficial effect. In the UK, dosing for depressive disorder starts at 25 $\mathrm{mg}$ /day and may be increased to up to $50 \mathrm{mg}$ /day. Regarding the incidence of depression, Kasper et al used a SIGH-SAD score of $\geq 16$, which may have resulted in an overestimate of the incidence of depression. This is to be seen in the context, and as previously stated, that the authors of the Cochrane Review set a SIGH-SAD cut-off score of $\geq 20$ a priori.

\section{Limitations}

There are some important limitations to this Cochrane Review. These include identifying only one eligible study, with a high attrition rate and employing a modified ITT analysis for the analysis of efficacy. Owing to the high attrition rate, it is unclear whether those who left the study could have developed depression and thus potentially altered the incidence rate in a significant way. Also, the setting does not reflect a real-world prevention experience with the participants being treated with agomelatine before winter starts and subsequently required to display stable remission for at least 2 months so as to be eligible for the study; therefore the generalisability of this study is poor.

\section{Research into prevention of $S A D$}

There is an evident lack of research on the topic of $\mathrm{SAD}$ prevention. Other Cochrane Reviews on SAD prevention but using the interventions of light therapy (Nussbaumer-Streit 2019b) and psychotherapy (Forneris 2019) also included only one study, which was at high risk of bias. However, a Cochrane Review on second-generation antidepressants in preventing SAD yielded more substantial results, demonstrating the effectiveness of 
bupropion extended release (XL) in preventing SAD but also highlighting a higher risk of harm associated with the medication (Gartlehner 2019).

\section{Conclusions}

On the basis of this Cochrane Review, no conclusion on the clinical significance of melatonergic agents in the prevention of $\mathrm{SAD}$ can be reached. NICE guidelines recommend management of seasonal depression using the same treatment pathway as for a major depressive episode. No advice is given on the prevention of SAD. Overall, it is unlikely that this review will influence clinical practice in the UK. Further research is required on this topic and clinical practice should be guided as always by the evidence available and patients' preferences, highlighting the benefits and risks of any intervention employed.

\section{Acknowledgements}

I thank Dr Riccardo De Giorgi (Department of Psychiatry, University of Oxford) for his advice on this project.

\section{Declaration of interest}

The views expressed here are the views of the author and not necessarily those of the National Health Service.

An ICMJE form is in the supplementary material, available online at https://doi.org/10.1192/bja. 2020.25 .

\section{References}

National Institute for Health and Care Excellence (2009) Depression in Adults: Recognition and Management (NICE Clinical Guideline CG90). NICE.

American Psychiatric Association (2013) Diagnostic and Statistical Manual of Mental Disorders (5th edn) (DSM-5). American Psychiatric Publishing.

Akram F, Jennings TB, Stiller JW, et al (2019) Mood worsening on days with high pollen counts is associated with a summer pattern of seasonality. Pteridines, 30: 133-41.

BMJ (2009) Management of seasonal affective disorder. Drug and Therapeutics Bulletin, 47: 128-32.

Cipriani A, Furukawa TA, Salanti G, et al (2018) Comparative efficacy and acceptability of 21 antidepressant drugs for the acute treatment of adults with major depressive disorder: a systematic review and network metaanalysis. Lancet, 391: 1357-66.

Forneris CA, Nussbaumer-Streit B, Morgan LC, et al (2019) Psychological therapies for preventing seasonal affective disorder. Cochrane Database of Systematic Reviews, 5: CD011270. Available from: https://doi.org/10. 1002/14651858.CD011270.pub3.

Gartlehner G, Nussbaumer-Streit B, Gaynes BN, et al (2019) Second-generation antidepressants for preventing seasonal affective disorder in adults. Cochrane Database of Systematic Reviews, 3: CD011268. Available from: https://doi.org/10.1002/14651858.CD011268.pub3.

Geoffroy PA, Bellivier F, Scott J, et al (2013) Bipolar disorder with seasonal pattern: clinical characteristics and gender influences. Chronobiology International, 30: 1101-7.

Hickie IB, Rogers NL (2011) Novel melatonin-based therapies: potential advances in the treatment of major depression. Lancet, 378: 621-31

Higgins JPT, Thomas J, Chandler J, et al (eds) (2019) Cochrane Handbook for Systematic Reviews of Interventions (Version 6.0, updated July 2019). Cochrane (www.training.cochrane.org/handbook).

Levitan RD (2007) The chronobiology and neurobiology of winter seasonal affective disorder. Dialogues in Clinical Neuroscience, 9: 315-24.

Lewy AJ, Emens JS, Songer JB, et al (2009) Winter Depression: Integrating mood, circadian rhythms, and the sleep/wake and light/dark cycles into a bio-psycho-social-environmental model. Sleep Medicine Clinics, 4: 285-99.

Magnusson A, Partonen T (2005) The diagnosis, symptomatology, and epidemiology of seasonal affective disorder. CNS Spectrums, 10: 625-34.

Monteleone P, Maj M (2008) The circadian basis of mood disorders: recent developments and treatment implications. Eur Neuropsychopharmacol, 18: 701-11.

Nussbaumer-Streit B, Greenblatt A, Kaminski-Hartenthaler A, et al (2019a) Melatonin and agomelatine for preventing seasonal affective disorder. Cochrane Database of Systematic Reviews, 6: CD011271. Available from: https://doi.org/10.1002/14651858.CD011271.pub3.

Nussbaumer-Streit B, Forneris CA, Morgan LC, et al (2019b) Light therapy for preventing seasonal affective disorder. Cochrane Database of Systematic Reviews, 3: CD011269. Available from: https://doi.org/10. 1002/14651858.CD011269.pub3.

Pjrek E, Winkler D, Konstantinidis A, et al (2007) Agomelatine in the treatment of seasonal affective disorder. Psychopharmacology, 190: 575-9.

Rosenthal NE, Sack DA, Gillin JC, et al (1984) Seasonal affective disorder: a description of the syndrome and preliminary findings with light therapy. Archives of General Psychiatry, 41: 72-80.

Sackett DL (1979) Bias in analytic research. Journal of Chronic Diseases, 32: 51-63.

Schünemann H, Brożek J, Guyatt G, et al (2013) Handbook for Grading the Quality of Evidence and the Strength of Recommendations Using the GRADE Approach (Updated October 2013). GRADE Working Group (https://gdt.gradepro.org/app/handbook/handbook.html).

Srinivasan V, De Berardis D, Shillcutt SD, et al (2012) Role of melatonin in mood disorders and the antidepressant effects of agomelatine. Expert Opinion on Investigational Drugs, 21: 1503-22.

Thompson C, Thompson S, Smith R (2004) Prevalence of seasonal affective disorder in primary care; a comparison of the Seasonal Health Questionnaire and the Seasonal Pattern Assessment Questionnaire. Journal of Affective Disorders, 78: 219-26.

Walker WH, Walton JC, Devries AC, et al (2020) Circadian rhythm disruption and mental health. Translational Psychiatry, 10: 28

World Health Organization (1992) The ICD-10 Classification of Mental and Behavioural Disorders: Clinical Descriptions and Diagnostic Guidelines. World Health Organization. 